\title{
Rectal Dose Is the Other Dosimetric Factor in Addition to Small Bowel for Prediction of Acute Diarrhea during Postoperative Whole-Pelvic Intensity-Modulated Radiotherapy in Gynecologic Patients
}

\author{
Eng-Yen Huang ${ }^{1,2,+}$, Yu-Ming Wang ${ }^{1,2,+}$, Shih-Chen Chang ${ }^{1}$, Shu-Yu Liu ${ }^{1}$ and Ming-Chung Chou ${ }^{3,4,5, *(D)}$ \\ 1 Department of Radiation Oncology, Kaohsiung Chang Gung Memorial Hospital, College of Medicine, \\ Chang Gung University, Kaohsiung 833, Taiwan; hey1200@adm.cgmh.org.tw (E.-Y.H.); \\ scorpion@cgmh.org.tw (Y.-M.W.); kenny25wed@cgmh.org.tw (S.-C.C.); jessica633@cgmh.org.tw (S.-Y.L.) \\ 2 School of Traditional Chinese Medicine, Chang Gung University, Kaohsiung 833, Taiwan \\ 3 Department of Medical Imaging and Radiological Sciences, Kaohsiung Medical University, \\ Kaohsiung 807, Taiwan \\ 4 Center for Big Data Research, Kaohsiung Medical University, Kaohsiung 807, Taiwan \\ 5 Department of Medical Research, Kaohsiung Medical University Hospital, Kaohsiung 807, Taiwan \\ * Correspondence: mcchou@kmu.edu.tw; Tel.: +886-7-312-1101; Fax: +886-7-311-3449 \\ + Equal contribution.
}

check for updates

Citation: Huang, E.-Y.; Wang, Y.-M.; Chang, S.-C.; Liu, S.-Y.; Chou, M.-C. Rectal Dose Is the Other Dosimetric Factor in Addition to Small Bowel for Prediction of Acute Diarrhea during Postoperative Whole-Pelvic Intensity-Modulated Radiotherapy in Gynecologic Patients. Cancers 2021, 13, 497. https://doi.org/10.3390/ cancers13030497

Academic Editors: Meritxell Arenas and Pierfrancesco Franco

Received: 28 December 2020

Accepted: 21 January 2021

Published: 28 January 2021

Publisher's Note: MDPI stays neutral with regard to jurisdictional claims in published maps and institutional affiliations.

Copyright: (c) 2021 by the authors. Licensee MDPI, Basel, Switzerland. This article is an open access article distributed under the terms and conditions of the Creative Commons Attribution (CC BY) license (https:/ / creativecommons.org/licenses/by/ $4.0 /)$.
Simple Summary: Although the small bowel volume effect for acute diarrhea during radiotherapy has been investigated, no study has reported the influence of rectal dose. We analyzed 108 patients undergoing intensity-modulated radiotherapy after hysterectomy. Acute diarrhea was defined as onset during radiotherapy based on Common Terminology Criteria for Adverse Events (CTCAE) version 3. Both small bowel and rectum dosimetric parameters affected Grade 2 to 3 diarrhea. The high-dose volume effects on the small bowel still play an important role in postoperative intensitymodulated radiotherapy. This is the first large cohort study to demonstrate the role of both IMRT dosimetric factors of the rectum and the small bowel in acute diarrhea in gynecological patients with a previous hysterectomy. A small bowel volume of $39.6 \mathrm{~Gy}<60 \mathrm{~mL}$ and a mean rectal dose of $<32.75$ Gy are suggested as constraints to treatment planning.

Abstract: We studied the association of rectal dose with acute diarrhea in patients with gynecologic malignancies undergoing whole-pelvic (WP) intensity-modulated radiotherapy (IMRT). From June 2006 to April 2019, 108 patients with previous hysterectomy who underwent WP IMRT were enrolled in this cohort study. WP irradiation of 39.6-45 Gy/22-25 fractions was initially delivered to the patients. Common Terminology Criteria for Adverse Events (CTCAE) version 3 was used to evaluate acute diarrhea during radiotherapy. Small bowel volume at different levels of isodose curves ( $\mathrm{Vn} \%)$ and mean rectal dose (MRD) were measured for statistical analysis. The multivariate analysis showed that the MRD $\geq 32.75 \mathrm{~Gy}(p=0.005)$ and small bowel volume of $100 \%$ prescribed $(\mathrm{V} 100 \%) \geq 60 \mathrm{~mL}$ $(p=0.008)$ were independent factors of Grade 2 or higher diarrhea. The cumulative incidence of Grade 2 or higher diarrhea at 39.6 Gy were $70.5 \%, 42.2 \%$, and $15.0 \%(p<0.001)$ in patients with both high (V100\% $\geq 60 \mathrm{~mL}$ and MRD $\geq 32.75 \mathrm{~Gy}$ ), either high, and both low volume-dose factors, respectively. Strict constraints for the rectum/small bowel or image-guided radiotherapy to reduce these doses are suggested.

Keywords: rectal dose; small bowel; IMRT; gynecologic malignancies; diarrhea

\section{Introduction}

Acute gastrointestinal (GI) toxicities are common side effects during pelvic radiotherapy. Although they are usually transient and reversible, consequential late effects may be 
troublesome to management in some studies. It is important to reduce the incidence and severity of GI toxicities as much as possible to improve patient quality of life. Symptoms of toxicity are nausea, vomiting, diarrhea, tenesmus, and abdominal cramping. Diarrhea is the most common symptom used for evaluating toxicity and is caused by radiation damage to the bowels, which leads to impairment of water absorption. Radiation-induced inflammation may cause hypermobility and further impairment of bowel function. The small bowel, colon, and rectum are commonly defined as organs at risk (OARs) in radiotherapy, and the excessive irradiation of these OARs could result in GI complications.

The effects of small bowel volume on acute radiation-induced GI toxicities have been well-studied in different diseases treated by pelvic radiotherapy. Although intensitymodulated radiotherapy (IMRT) can reduce GI complications [1-3], studies of the small bowel volume effect have seldom been investigated in patients undergoing IMRT. Wang et al. noted the consequential effect of acute diarrhea and late rectal toxicity [4]. The conclusion implies the role of the rectum in acute diarrhea during pelvic irradiation, although this has seldom been investigated. A suitable model for studying the dosimetric effects of the small bowel and rectum is gynecological malignancies because partial volumes of the small bowel and rectum are irradiated. Therefore, the aim of the present study was to address the dosimetric effects of the small bowel and rectum on acute diarrhea in patients with gynecological malignancies who underwent IMRT.

\section{Materials and Methods}

\subsection{Patients and Radiotherapy}

We established a cohort to investigate the correlation between radiation-induced GI toxicities and dosimetry in gynecological malignancies since 2003. From June 2006 to April 2019, 108 patients who received previous hysterectomy for gynecological malignancies and underwent whole-pelvic IMRT were reviewed in the cohort. Before radiotherapy, all patients underwent immobilization in the supine position using a thermoplastic cast and CT-simulation. The patients were encouraged to avoid emptying their bladders before and during the simulations. Rectum emptying was encouraged but not mandatory. Noncontrast CT images with a $5 \mathrm{~mm}$ slice thickness were obtained. Contouring of the clinical target volume (CTV) included the vagina, external iliac, internal iliac, and common iliac lymph nodes. Planning target volume (PTV) was an extension of CTV plus $10 \mathrm{~mm}$ in all directions based on our setup error data. The bowel loops of the small intestine, bladder, femoral heads, and rectum were contoured for dosimetric calculation using the Pinnacle treatment planning system (ADAC Laboratories, Milpitas, CA, USA). The rectum was delineated from the level of the anus to the sigmoid flexure. Slice by slice, we tracked the colon from the rectosigmoid colon to the descending colon and the ileocecal junction to the ascending colon. The remaining bowel loops we contoured were defined as the small intestine. The separate loops of visible small bowel were delineated from its lowest extent to $2 \mathrm{~cm}$ above the CTV. The constraints of V40 Gy were $<30 \%$ for the small bowel, $<60 \%$ for the rectum, $<70 \%$ for the bladder, and $<50 \%$ for the femoral heads. In general, 7 fields $\left(30^{\circ}\right.$, $80^{\circ}, 130^{\circ}, 180^{\circ}, 230^{\circ}, 280^{\circ}$, and $330^{\circ}$ ) of IMRT were arranged. No image guidance during daily irradiation was performed. The step-and-shoot technique was used to perform beam delivery: the whole-pelvic dose ranged from 39.6-45 Gy. Some patients received a low pelvic or local boost after whole-pelvic IMRT. V10\% was defined as the small bowel volume covered at $10 \%$ of the isodose curve, and V20\% to $\mathrm{V} 100 \%$ were also recorded for analysis. Therefore, the V10\% V100\% were absolute small bowel volumes (mL) at relative doses. If the $\mathrm{V} 100 \%$ was $80 \mathrm{~mL}$ in a patient undergoing a $39.6 \mathrm{~Gy}$ whole-pelvic $\mathrm{RT}$, then the V100\% was still $80 \mathrm{~mL}$ in the same patient when the prescribed dose of whole-pelvic RT was increased to $45 \mathrm{~Gy}$. In addition to the small bowel, the mean rectal dose (MRD) was recorded for comparison. We used Common Terminology Criteria for Adverse Events (CTCAE) version 3 for diarrhea grading. We evaluated patients weekly and recorded the onset time of any grade of diarrhea in the chart during radiotherapy. Because medication 
may affect the grading of acute diarrhea, the principle of medical management of acute diarrhea was based on a previous study [5].

\subsection{Statistics}

An independent t-test was used to compare patients with different grades of GI toxicity. A receiver operating characteristic (ROC) curve was used to determine the optimal cut-off of dosimetric data for acute diarrhea, and the area under the curve (AUC) was calculated for comparisons. Similar to the survival analysis, we used the Kaplan-Meier method as an actuarial analysis for calculating the cumulative incidence of acute GI toxicity, and the onset dose of toxicity was recorded at each grade. A log-rank test was used to compare the significance between different groups. The end dose in the evaluation of diarrhea grade was $39.6 \mathrm{~Gy}$ because not all patients underwent a whole-pelvic dose of $45 \mathrm{~Gy}$. Therefore, we set the whole-pelvic dose of 39.6 Gy as 100\% standardized for dosimetry of the small bowel and the rectum. However, in patients without certain grades of diarrhea, the event of diarrhea was censored, and the final dose was 39.6 Gy. For example, the Grade 1 and 2 doses were 18 and $36 \mathrm{~Gy}$, respectively, in one patient. No Grade 3 diarrhea was noted, and the event of Grade 1, 2, or 3 diarrhea was treated as uncensored at $18 \mathrm{~Gy}$, uncensored at $36 \mathrm{~Gy}$, and censored at $39.6 \mathrm{~Gy}$, respectively. Once toxicity-related interruption was noted; the event of diarrhea was uncensored at the event dose. For example, Grade 3 diarrhea was noted in a patient at $28.8 \mathrm{~Gy}$, and treatment interruption was allowed. The event of Grade 3 was treated as uncensored at $28.8 \mathrm{~Gy}$. Therefore, the influence of time courses on the occurrence of acute diarrhea was minimized as well as survival analysis.

A multivariate analysis was performed using the Cox regression model with the forward procedure. Age, body mass index (BMI), and dosimetric data were treated as continuous variables. Concurrent chemoradiotherapy (CCRT), diabetes, and hypertension were treated as a binary variable. After determining the optimal cut-off using the ROC curve, a Cox regression model using categorical variables was used to confirm the dosimetric significance. The result was considered significant if $p<0.05$.

\section{Results}

The characteristics of 108 patients are shown in Table 1. About two-thirds of the patients were diagnosed with endometrial cancer. The dose per fraction of IMRT was 1.8 Gy in all cases. After a whole-pelvic IMRT of up to 39.6 45 Gy, 91 patients received an additional IMRT boost of 1.8-25.2 Gy to the low pelvic, vagina, or gross tumor site. The cumulative incidence of Grade 2 or greater diarrhea at 39.6 Gy was $48.3 \%$. The cumulative incidence of Grade 3 diarrhea at 39.6 Gy was $18.8 \%$.

Table 1. Characteristics of patients $(n=108)$.

\begin{tabular}{ll}
\hline Characteristics & Mean \pm SEM or Number $(\%)$ \\
\hline Age $($ years $)$ & $53.6 \pm 1.0$ \\
BMI $\left(\mathrm{kg} / \mathrm{m}^{2}\right)$ & $24.7 \pm 0.4$ \\
Diabetes & \\
No & $96(88.9 \%)$ \\
Yes & $12(11.1 \%)$ \\
Hypertension & \\
No & $85(78.7 \%)$ \\
Yes & $23(21.3 \%)$ \\
Disease & \\
Cervical cancer & $32(29.6 \%)$ \\
Endometrial cancer & $72(66.7 \%)$ \\
Uterine sarcoma & $4(3.7 \%)$ \\
CCRT & $84(77.8 \%)$ \\
No & $24(22.2 \%)$ \\
Yes & \\
\hline
\end{tabular}


Table 1. Cont.

\begin{tabular}{ll}
\hline Characteristics & Mean \pm SEM or Number (\%) \\
\hline IMRT dose & \\
WP $<39.6$ Gy & $(4.6 \%)$ \\
WP 39.6 Gy & $4(3.7 \%)$ \\
No boost & $1(0.9 \%)$ \\
LP 41.4 Gy & $43(39.8 \%)$ \\
LP 45 Gy & $39(36.1 \%)$ \\
LP 50.4 Gy & $6(5.6 \%)$ \\
Local boost to 50.4-64.8 Gy & \\
WP 45 Gy & $8(7.4 \%)$ \\
No boost & $1(0.9 \%)$ \\
LP 50.4 Gy & $1(0.9 \%)$ \\
Local boost to 64.8 Gy & \\
Diarrhea & $21(19.4 \%)$ \\
Grade 0 & $35(32.4 \%)$ \\
Grade 1 & $32(29.6 \%)$ \\
Grade 2 & $20(18.5 \%)$ \\
Grade 3 & $0(0 \%)$ \\
Grade 4 &
\end{tabular}

Abbreviations: $\mathrm{BMI}=$ Body mass index; CCRT = Concurrent chemoradiotheray; $\mathrm{LP}=$ Low pelvis; $\mathrm{SEM}$ = Standard error of mean; $\mathrm{WP}=$ Whole pelvis.

\subsection{Dosimetric Data between Acute Grade 0-1 and Grade 2-3 Diarrhea}

We compared V10\% to V100\% and MRD between patients with Grade 0-1 and Grade 2-3 toxicity. Significant differences were noted between patients with Grade $0-1$ and Grade $2-3$ toxicity at V80\% $(p=0.044), \mathrm{V} 90 \%(p=0.029), \mathrm{V} 100 \%(p=0.020)$, and $\mathrm{MRD}(p=0.006)$ (Table 2). We analyzed V10\% to V100\% and MRD for the prediction of Grade 2-3 toxicity using ROC curve analysis. Significant roles of V80\% (AUC $=0.618)$, V90\% (AUC = 0.629), $\mathrm{V} 100 \%(\mathrm{AUC}=0.629)$, and MRD $(\mathrm{AUC}=0.627)$ for the prediction of Grade $2-3$ toxicity were also noted (Table 3). Moreover, the optimal cut-off was V100\% $=60 \mathrm{~mL}$ for small bowel volume (sensitivity $65.4 \%$ and specificity $64.3 \%$ ) and was MRD $=32.75$ Gy for the rectum (sensitivity $86.5 \%$ and specificity $43.9 \%$ ) as determined by the ROC curve analysis. Based on these analyses, we selected V100\% $=60 \mathrm{~mL}$ and MRD $=32.75 \mathrm{~Gy}$ as the small bowel and rectum dosimetric factors, respectively.

Table 2. Dosimetric data between Grade 0-1 and Grade 2-3 diarrhea.

\begin{tabular}{cccc}
\hline Parameter & Grade $\mathbf{0 - 1}$ & Grade 2-3 & $p$-Value \\
\hline V10\% (mL) & $434 \pm 28$ & $436 \pm 26$ & 0.987 \\
V20\% (mL) & $405 \pm 26$ & $415 \pm 25$ & 0.763 \\
V30\% (mL) & $373 \pm 24$ & $394 \pm 24$ & 0.543 \\
V40\% (mL) & $328 \pm 20$ & $361 \pm 22$ & 0.283 \\
V50\% (mL) & $281 \pm 20$ & $311 \pm 20$ & 0.286 \\
V60\% (mL) & $235 \pm 17$ & $270 \pm 19$ & 0.165 \\
V70\% (mL) & $192 \pm 15$ & $232 \pm 17$ & 0.076 \\
V80\% (mL) & $150 \pm 13$ & $189 \pm 15$ & 0.044 \\
V90\% (mL) & $106 \pm 10$ & $138 \pm 11$ & 0.029 \\
V100\% (mL) & $61 \pm 6$ & $83 \pm 7$ & 0.020 \\
MRD (Gy) & $33.57 \pm 0.58$ & $35.58 \pm 0.31$ & 0.006
\end{tabular}


Table 3. ROC curve for Grade 2-3 diarrhea.

\begin{tabular}{cccc}
\hline Parameter & AUC & $\mathbf{9 5 \%}$ CI & $p$-Value \\
\hline V10\% & $0.509 \pm 0.056$ & $0.399-0.619$ & 0.873 \\
V20\% & $0.521 \pm 0.056$ & $0.411-0.630$ & 0.712 \\
V30\% & $0.539 \pm 0.056$ & $0.430-0.648$ & 0.483 \\
V40\% & $0.558 \pm 0.056$ & $0.449-0.666$ & 0.303 \\
V50\% & $0.570 \pm 0.055$ & $0.462-0.679$ & 0.210 \\
V60\% & $0.583 \pm 0.055$ & $0.475-0.691$ & 0.136 \\
V70\% & $0.602 \pm 0.055$ & $0.494-0.709$ & 0.068 \\
V80\% & $0.618 \pm 0.055$ & $0.511-0.725$ & 0.035 \\
V90\% & $0.629 \pm 0.054$ & $0.523-0.736$ & 0.020 \\
V100\% & $0.629 \pm 0.054$ & $0.523-0.735$ & 0.021 \\
Rectal dose & $0.627 \pm 0.054$ & $0.522-0.733$ & 0.023 \\
\hline
\end{tabular}

Abbreviations: $\mathrm{AUC}=$ area under the curve; $\mathrm{CI}=$ confidence interval.

\subsection{Cumulative Incidence of Acute Grade 2-3 Diarrhea}

The Kaplan-Meier analysis showed that the cumulative incidence of Grade 2-3 diarrhea at $39.6 \mathrm{~Gy}$ in patients with $<60 \mathrm{~mL}$ and $\geq 60 \mathrm{~mL}$ was $33.3 \%$ and $63.4 \%(p=0.001)$, respectively (Figure 1A). The cumulative incidence of Grade 2-3 diarrhea at $39.6 \mathrm{~Gy}$ in patients with MRD $<32.75 \mathrm{~Gy}$ and $\geq 32.75$ Gy was $22.6 \%$ and $58.8(p=0.001)$, respectively (Figure 1B).
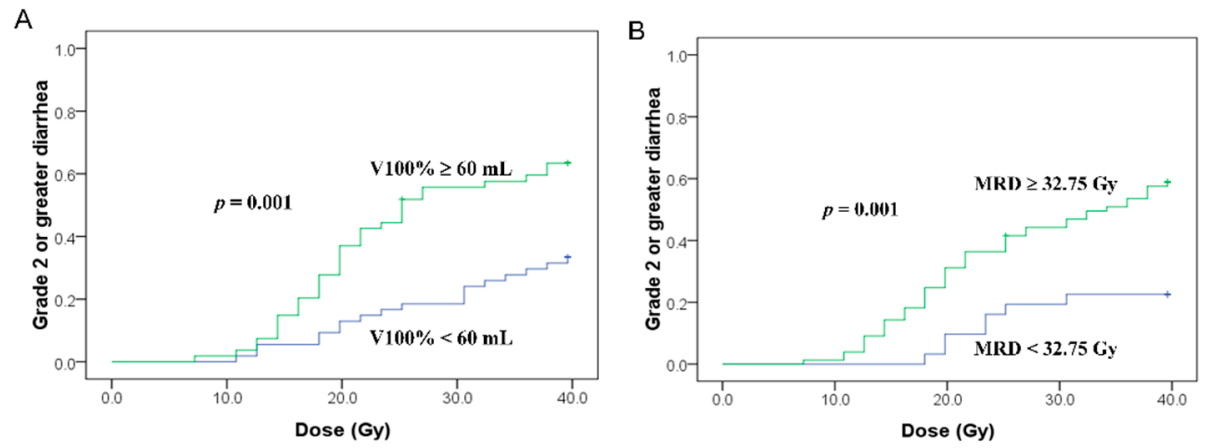

Figure 1. Small bowel volume effect (A) and rectal dose effect (B) for Grade 2 or greater diarrhea. The horizontal axis is the cumulative prescribed dose to the whole-pelvic target.

3.3. Multivariate Analysis of Dosimetric and Non-Dosimetric Data for Acute Grade 2-3 Diarrhea

The multivariate analysis (Table 4$)$ showed that V100\% $(p=0.005)$ and $\operatorname{MRD}(p=0.008)$ were significant factors for Grade 2-3 diarrhea, while CCRT had a statistical trend $(p=0.053)$. V100\% $(p=0.006)$ remained a significant factor for Grade 3 diarrhea.

Table 4. Multivariate analyses for Grade 2-3 diarrhea.

\begin{tabular}{ccccccc}
\hline Parameter & \multicolumn{3}{c}{ Grade 2-3 } & \multicolumn{3}{c}{ Grade 3 } \\
\hline & HR & $\mathbf{9 5 \%}$ CI & $p$-Value & HR & $\mathbf{9 5 \% ~ C I ~}$ & $p$-Value \\
\hline Diabetes & - & - & 0.884 & - & - & 0.666 \\
Hypertension & - & - & 0.284 & - & - & 0.724 \\
CCRT & - & - & 0.053 & - & - & 0.106 \\
V100\% $>60$ & 2.286 & $1.282 \sim 4.075$ & 0.005 & 4.622 & $1.544 \sim 1.3833$ & 0.006 \\
mL & & & & & - & 0.263 \\
MRD $>32.75$ & 2.980 & $1.336 \sim 6.648$ & 0.008 & - & -
\end{tabular}

Abbreviations: $\mathrm{MRD}=$ mean rectal dose; CCRT = concurrent chemoradiotheray; HR = hazard ratio $\mathrm{CI}=$ confidence interval. 


\subsection{Combination of Small Bowel and Rectum Dosimetry for Prediction of Diarrhea}

The combination of small bowel and rectum dosimetry revealed a better AUC in the ROC curve analysis (Figure 2). The AUC was $0.627(p=0.023), 0.629(p=0.021)$, and $0.714(p<0.001)$ for rectum, small bowel, and combination in Grade 2 or greater diarrhea, respectively. The corresponding AUC was $0.628(p=0.074), 0.660(p=0.026)$, and 0.701 $(p=0.005)$ for Grade 3 diarrhea.

A

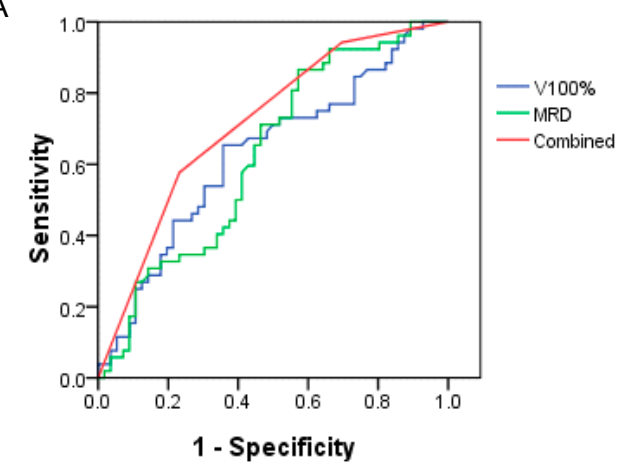

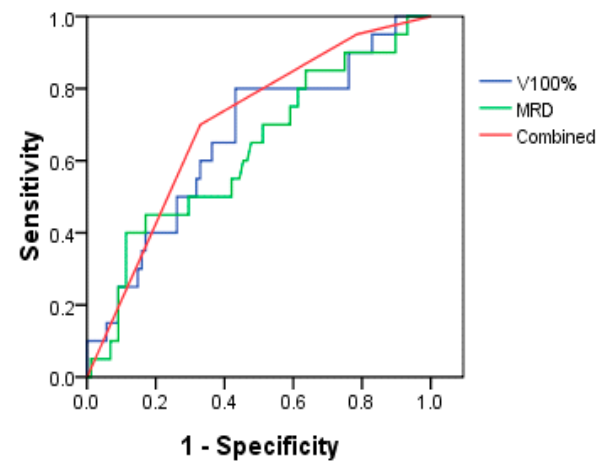

Figure 2. The ROC curve for combination of small bowel volume effect and rectal dose effect on Grade 2 or greater (A) and Grade 3 (B) diarrhea.

Cumulative rates of Grade 2 or greater diarrhea at 39.6 Gy were $15.0 \%, 42.2 \%$, and $70.5 \%$ in low (V100\% < $60 \mathrm{~mL}$ and MRD < 32.75 Gy), low /high (V100\% < $60 \mathrm{~mL}$ and $\mathrm{MRD} \geq 32.75 \mathrm{~Gy}$, or $\mathrm{V} 100 \% \geq 60 \mathrm{~mL}$ and $\mathrm{MRD}<32.75 \mathrm{~Gy})$, and both high $(\mathrm{V} 100 \% \geq 60 \mathrm{~mL}$ and MRD $\geq 32.75 \mathrm{~Gy}$ ) groups $(p<0.001)$ (Figure 3A), while the corresponding rates of Grade 3 diarrhea were $5.0 \%, 11.4 \%$, and $32.8 \%(p=0.005)$ (Figure $3 \mathrm{~B})$.
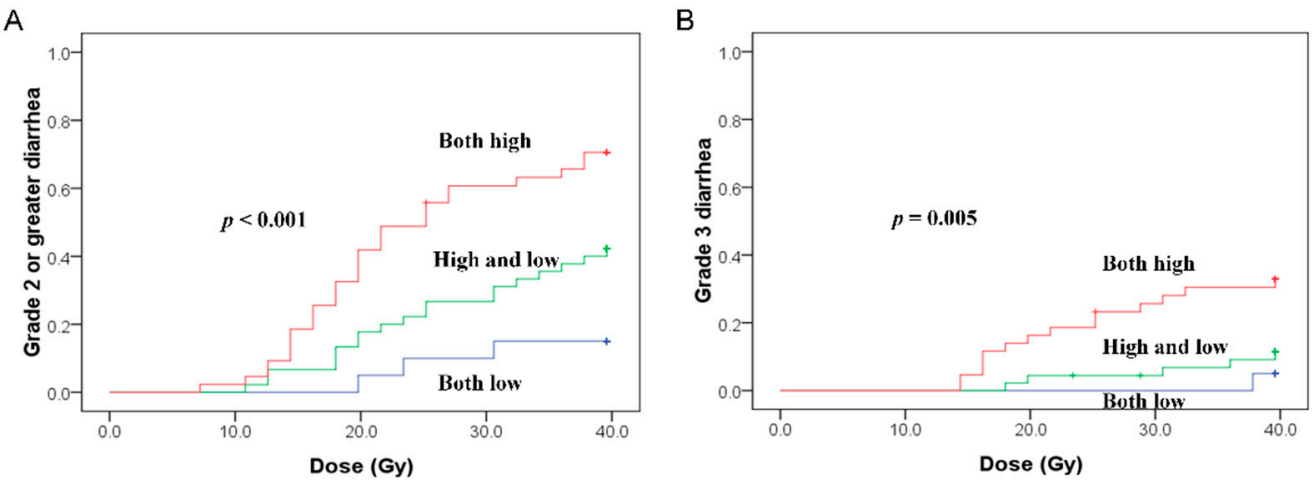

Figure 3. The combination of the small bowel volume effect and rectal dose effect on Grade 2 or greater (A) and Grade 3 (B) diarrhea. Both high indicates the patient group with $\mathrm{V} 100 \% \geq 60 \mathrm{~mL}$ and $\mathrm{MRD} \geq 32.75 \mathrm{~Gy}$. Either high indicates the patient group with $\mathrm{V} 100 \% \geq 60 \mathrm{~mL}$ and $\mathrm{MRD}<32.75 \mathrm{~Gy}$ or $\mathrm{V} 100 \%<60 \mathrm{~mL}$ and MRD $\geq 32.75 \mathrm{~Gy}$. Both low indicates the patient group with $\mathrm{V} 100 \%<60 \mathrm{~mL}$ and $\mathrm{MRD}<32.75 \mathrm{~Gy}$.

\section{Discussion}

To date, there are few studies about the effects of small bowel volume in gynecological IMRT patients, and only one study reported rectal dosimetry for acute GI toxicity. Roeske et al. first noted a high-dose (100\%) small bowel volume effect in whole-pelvic IMRT patients $(n=50)$, the majority of whom had gynecological malignancies [6], and approximately two-thirds had received hysterectomies. Rectal dosimetry (range 35-49 Gy) was not a significant factor in acute GI toxicity. Isohashi et al. also found a high-dose (90\%) small bowel volume effect in whole-pelvic cervical cancer patients $(n=62)$ with radical hysterectomies [7]. Isohashi et al. demonstrated that IMRT $(n=30)$ significantly decreased Grade 2 or higher acute GI complications (63\%) in comparison to three-dimensional confor- 
mal radiotherapy (3D-CRT) $(n=32)(94 \%)(p<0.01)$. Furthermore, high-dose (V45 Gy) but not low-dose (V15 Gy) small bowel volumes were significantly smaller in the IMRT group. However, this study did not analyze the small bowel volume effect separately in IMRT and 3D-CRT patients. The IMRT data of acute GI complications (Grade $\geq 2$ : 63\%; Grade 3: 20\%) were similar to our data (Grade $\geq 2: 48.1 \%$; Grade 3: $18.5 \%$ ). Chi et al. found a high-dose (V45 Gy) small bowel volume effect using IMRT in patients $(n=32)$ with endometrial cancer [8]. Furthermore, Li et al. noted that 39\% of IMRT patients $(n=23)$ had Grade 2-3 diarrhea, but no significant small bowel volume effects [9]. The literature reviews of acute GI toxicity in patients undergoing IMRT pelvic radiotherapy are listed in Table 5.

Table 5. Literature review of acute GI toxicity in patients undergoing IMRT pelvic radiotherapy.

\begin{tabular}{|c|c|c|c|c|c|c|c|c|}
\hline Author (Reference) & $n$ & Disease & OP & Grade 2 & $\begin{array}{l}\text { Large } \\
\text { Fields }\end{array}$ & Balloon & $\begin{array}{c}\text { Small Bowel } \\
\text { Volume }\end{array}$ & Rectum \\
\hline Roeske et al. [6] & 50 & GYN & $68 \%$ & $28 \%$ & $100 \%$ & $(-)$ & V45 Gy & No effect \\
\hline Isohashi et al. [7] & 30 & cervix & $100 \%$ & $63 \%$ & $100 \%$ & $(-)$ & NA & NA \\
\hline Chi et al. [8] & 32 & GYN & $100 \%$ & $34 \%$ & $100 \%$ & $(-)$ & V45 Gy & NA \\
\hline Li et al. [9] & 23 & GYN & $100 \%$ & $39 \%$ & $100 \%$ & $(-)$ & No effect & NA \\
\hline Wu et al. [10] & 28 & GYN & $100 \%$ & $18 \%$ & $100 \%$ & $(+)$ & NA & NA \\
\hline Olsen et al. [11] & 52 & anal & $0 \%$ & $67 \%$ & $100 \%$ & $(-)$ & V25-35 Gy & NA \\
\hline Samuelian et al. [12] & 31 & rectal & $19 \%$ & $32 \%$ & $100 \%$ & $(-)$ & NA & NA \\
\hline Deville et al. [13] & 30 & prostate & $0 \%$ & $50 \%$ & $100 \%$ & $(-)$ & NA & No effect \\
\hline Deville et al. [14] & 67 & prostate & $100 \%$ & $46 \%$ & $54 \%$ & $(-)$ & NA & Dmin \\
\hline Teh et al. [15] & 100 & prostate & $0 \%$ & $6 \%$ & $0 \%$ & $(+)$ & NA & No effect \\
\hline Deville et al. [16] & 100 & prostate & $0 \%$ & $8 \%$ & $0 \%$ & $(+)$ & NA & MRD \\
\hline Klopp et al. [17] & 122 & GYN & $100 \%$ & $26 \%$ & $100 \%$ & $(-)$ & NA & NA \\
\hline Present study & 108 & GYN & $100 \%$ & $48 \%$ & $100 \%$ & $(-)$ & V39.6 Gy & MRD \\
\hline
\end{tabular}

Abbreviations: $\mathrm{OP}=$ operation; $\mathrm{MRD}=$ mean rectal dose; $\mathrm{NA}=$ not applicable.

The importance of the small bowel in acute GI toxicity could be emphasized through the comparison between whole-pelvic and prostate-only irradiation for prostate cancer $[13,14]$. Therefore, the higher incidence of acute Grade 2 or greater GI toxicity is considered to be caused by increased irradiation of the small bowel in the whole-pelvic patient group. In terms of excluding confounding factors in rectum irradiation, rectal cancer using preoperative CCRT is considered to be an appropriate disease model since the entire rectum is irradiated. Therefore, the incidence of acute GI toxicity is higher, and Grade 3 toxicity can be easily studied [18]. To date, a small number of studies have examined the small bowel volume effect using IMRT for rectal and anal cancer, in which the majority of patients received preoperative or definitive radiotherapy. Arbea et al. found that V10, V15, and V50 Gy of the small bowel were predictors of acute Grade 3 diarrhea [19], whereas Samuelian et al. noted that IMRT could reduce acute GI toxicity in rectal cancer $(n=92)$ [12]; however, dosimetric analysis was not performed in these studies. Xu et al. noted no small bowel volume effect [20], whereas in a study involving definitive IMRT for anal cancer $(n=52)$, Olsen et al. demonstrated a low-dose (V25-V40 Gy) small bowel volume effect [11]. This finding agrees with the results of Huang et al. [5], which demonstrated a low-dose small bowel volume effect in patients without prior abdominal surgery.

In addition to the small bowel, the importance of the rectum in acute radiation-induced GI toxicity has been proposed in patients with gynecological malignancies, and this was a specific aim of the present investigation. Although there are few studies that have examined the role of the rectum in acute radiation-induced GI toxicity in gynecological patients, radiotherapy of pelvic lesions, with an effort to spare the rectum, is a suitable disease model to support a rectal effect.

The most common disease in which to evaluate the most appropriate rectal dose for acute GI toxicity is prostate cancer, in which only the prostate is irradiated, in order to exclude the small bowel effect. In IMRT cases, $13 \%$ of patients had Grade 2 or greater toxic- 
ity [13], while $29 \%$ of patients without a rectal balloon had Grade 2 or greater toxicity [14]; correspondingly, $6 \%$ [15] and 8\% [16] of patients with a rectal balloon had Grade 2.

Rectal manipulation has also demonstrated the importance of the rectum in acute radiation-induced GI toxicity. Wu et al. hypothesized that the use of a rectal balloon could reduce acute and chronic toxicity in patients receiving IMRT for postoperative gynecological malignancies [10]. No small bowel dosimetry was analyzed in this study, and the mean percentage of rectal V30 and V40 Gy was $65.1 \%$ and $39.3 \%$, respectively. The MRD was expected to be $30-40 \mathrm{~Gy}$, which was similar to the results of our study. Further studies that correlate with rectal dosimetry and acute GI toxicity in patients with prostate cancer can provide stronger evidence for clinical practice. Peeters et al. noted a rectal wall volume effect ( $\mathrm{mL}$ and $\%$ at 30, 35, and $65 \mathrm{~Gy}$ ) and MRD [21]. Furthermore, a linear trend of $40 \mathrm{~Gy}$ rectal volume (\%) was demonstrated for acute GI side effects [22]. Dias et al. noted a $25 \%$ and $40 \%$ rectal volume effect in acute Grade 2 or greater GI toxicity, respectively [23]; Deville et al. demonstrated that infield MRD (Gy), V30 (\%), and V40 (\%) were important rectal dose parameters [16]. Teh et al. noted that the MRD of Grade $0-1$ and Grade 2 were 35.1-35.7 and 38.4 Gy, respectively [15]; this is similar to the difference between Grade 0-1 (33.57 Gy) and Grade 2-3 (35.58 Gy) diarrhea demonstrated in the present study. Based on these studies and the present results, we think that a reduction in rectal dose/volume could result in a decrease in Grade 2 or greater GI toxicity.

The main technique for examining dosimetric effects on acute GI toxicity of the small bowel and rectum is 3D-CRT [18]. In the era of IMRT, a reduction in small bowel and rectum irradiation can be achieved by setting strict dose constraints. Although there have been some clinical data comparison of 3D-CRT and IMRT, no dosimetric data were shown in these studies, and IMRT always limits the small bowel and rectum dose. It is reasonable to hypothesize that IMRT could be used to reduce acute GI toxicity through reduction in the small bowel and rectum dose. In addition, image-guided radiotherapy (IGRT) is a more aggressive technique that can be used to reduce the dose as a result of reducing PTV and OAR exposure of the small bowel [24] and rectum [25].

The current study included a large number of patients undergoing IMRT to study the dosimetric factors of acute radiation-induced GI toxicity (Table 5) in both the small bowel and rectum, which manifests as diarrhea. We used cumulative but not crude incidence for diarrhea, as it is predictable at certain doses during radiotherapy. Therefore, we recorded the onset dose of any grade of diarrhea and the dosimetry correlation to provide adequate information. The distinction between acute and late diarrhea would be helpful in the assessment of other data. Our evaluation time (weekly during RT) was similar to radiation therapy oncology group (RTOG) 1203, the IMRT pelvic radiation for post-operative treatment of endometrial and cervical cancer (TIME-C) trial (weeks 3 and 5) [17].

The limitation of the present study is the inability to perform rectum preparation at each treatment since the medication used for preparation interferes with the scoring of acute GI toxicity. The best model for this application is prostate stereotactic body radiotherapy (SBRT) since the appearance of acute GI toxicity is usually noted after the completion of SBRT (16\% of during SBRT to 57\% of 1 week from the end of SBRT) [26,27]. Regardless of whether the volume and position of the small bowel and rectum volume vary at each fraction of radiotherapy, the data still show a significant dosimetric correlation to clinical symptoms, in line with the results of a number of related studies. In gynecological patients, the dose of both the small bowel and rectum can be controlled using IMRT and IGRT. The cumulative incidence of Grade 2 or greater diarrhea during whole-pelvic irradiation in the both low-dose (V100\% and MRD) group was below 20\%. Modern advanced techniques such as proton therapy can be used to spare the GI tract [28] and may further reduce acute GI toxicity; thus, additional techniques warrant study in the future. 


\title{
5. Conclusions
}

In addition to the small bowel, the rectal dose is the other dosimetric factor involved in acute GI toxicity during whole-pelvic IMRT. Reducing the dose and volume of irradiation of these OARs can decrease the incidence of GI toxicity.

Author Contributions: E.-Y.H., Y.-M.W. and M.-C.C. designed the study. E.-Y.H., Y.-M.W., S.-C.C. and S.-Y.L. performed the data collection. E.-Y.H., Y.-M.W. and S.-C.C. performed the statistical analysis. E.-Y.H. and Y.-M.W. wrote the original draft. M.-C.C. supervised the project. All authors helped to revise and edit the manuscript through critical feedback. All authors have read and agreed to the published version of the manuscript.

Funding: This work was partially funded by a grant of the Chang-Gung Medical Research Project (CMRPG871101).

Institutional Review Board Statement: The study was conducted according to the guidelines of the Declaration of Helsinki and approved by the Institutional Review Board of Chang Gung Memorial Hospital (201701449B0).

Informed Consent Statement: Informed consent was obtained from all subjects involved in the study.

Data Availability Statement: The data presented in this study are available on request from the corresponding author. The data are not publicly available due to the nature of this research, participants of this study did not agree for their data to be shared publicly.

Conflicts of Interest: The authors declare no conflict of interest.

\begin{abstract}
Abbreviations
Radiation therapy (RT); Intensity-modulated radiotherapy (IMRT); Planned target volume (PTV); Clinical target volume (CTV); Gray (Gy); Gynecology (GYN); Common Terminology Criteria for Adverse Events (CTCAE); gastrointestinal (GI); mean rectal dose (MRD); Concurrent chemoradiotheray (CCRT); Whole pelvis (WP); Low pelvis (LP).
\end{abstract}

\section{References}

1. Chen, M.-F.; Tseng, C.-J.; Tseng, C.-C.; Kuo, Y.-C.; Yu, C.-Y.; Chen, W.-C. Clinical outcome in posthysterectomy cervical cancer patients treated with concurrent cisplatin and intensity-modulated pelvic radiotherapy: Comparison with conventional radiotherapy. Int. J. Radiat. Oncol. Biol. Phys. 2007, 67, 1438-1444. [CrossRef] [PubMed]

2. Ferrigno, R.; Santos, A.; Martins, L.C.; Weltman, E.; Chen, M.J.; Sakuraba, R.; Lopes, C.P.; Cruz, J.C. Comparison of conformal and intensity modulated radiation therapy techniques for treatment of pelvic tumors. Analysis of acute toxicity. Radiat. Oncol. 2010, 5, 117. [CrossRef] [PubMed]

3. Kwak, Y.K.; Lee, S.W.; Kay, C.S.; Park, H.H. Intensity-modulated radiotherapy reduces gastrointestinal toxicity in pelvic ra-diation therapy with moderate dose. PLoS ONE 2017, 12, e0183339. [CrossRef] [PubMed]

4. Wang, C.-J.; Leung, S.-W.; Chen, H.-C.; Sun, L.-M.; Fang, F.-M.; Huang, E.-Y.; Hsiung, C.-Y.; Changchien, C.-C. The correla-tion of acute toxicity and late rectal injury in radiotherapy for cervical carcinoma: Evidence suggestive of consequential late effect (CQLE). Int. J. Radiat. Oncol. Biol. Phys. 1998, 40, 85-91. [CrossRef]

5. Huang, E.-Y.; Sung, C.-C.; Ko, S.-F.; Wang, C.-J.; Yang, K.-D. The different volume effects of small-bowel toxicity during pel-vic irradiation between gynecologic patients with and without abdominal surgery: A prospective study with computed to-mography-based dosimetry. Int. J. Radiat. Oncol. Biol. Phys. 2007, 69, 732-739. [CrossRef]

6. Roeske, J.C.; Bonta, D.; Mell, L.K.; Lujan, A.E.; Mundt, A.J. A dosimetric analysis of acute gastrointestinal toxicity in women receiving intensity-modulated whole-pelvic radiation therapy. Radiother. Oncol. 2003, 69, 201-207. [CrossRef]

7. Isohashi, F.; Mabuchi, S.; Yoshioka, Y.; Seo, Y.; Suzuki, O.; Tamari, K.; Yamashita, M.; Unno, H.; Kinose, Y.; Kozasa, K.; et al. Intensity-modulated radiation therapy versus three-dimensional conformal radia-tion therapy with concurrent nedaplatin-based chemotherapy after radical hysterectomy for uterine cervical cancer: Compar-ison of outcomes, complications, and dose-volume histogram parameters. Radiat. Oncol. 2015, 10, 180.

8. Chi, A.; Nguyen, N.P.; Xu, J.; Ji, M.; Tang, J.; Jin, J.; Ong, E.S.; Welsh, J.S. Correlation of three different approaches of small bowel delineation and acute lower gastrointestinal toxicity in adjuvant pelvic intensity-modulated radiation therapy for en-dometrial cancer. Technol. Cancer Res. Treat. 2012, 11, 353-359. [CrossRef]

9. Li, Q.; Chen, J.; Zhu, B.; Jiang, M.; Liu, W.; Lu, E.; Liu, Q.-L. Dose Volume Effect of Acute Diarrhea in Post-Operative Radiation for Gynecologic Cancer. Rev. Investig. Clin 2017, 69. [CrossRef] 
10. Wu, C.-C.; Wuu, Y.-R.; Yanagihara, T.; Jani, A.; Xanthopoulos, E.P.; Tiwari, A.; Wright, J.D.; Burke, W.M.; Hou, J.Y.; Tergas, A.I.; et al. Rectal balloon use limits vaginal displacement, rectal dose, and rectal toxicity in patients receiving IMRT for postoperative gynecological malignancies. Med. Dosim. 2018, 43, 23-29. [CrossRef]

11. Olsen, J.R.; Moughan, J.; Myerson, R.; Abitbol, A.; Doncals, D.E.; Johnson, D.; Schefter, T.E.; Chen, Y.; Fisher, B.; Michalski, J.; et al. Predictors of Radiation Therapy-Related Gastrointestinal Toxicity From Anal Cancer Dose-Painted Intensity Modulated Radiation Therapy: Secondary Analysis of NRG Oncology RTOG. Int. J. Radiat. Oncol. 2017, 98, 400-408. [CrossRef] [PubMed]

12. Samuelian, J.M.; Callister, M.D.; Ashman, J.B.; Young-Fadok, T.M.; Borad, M.J.; Gunderson, L.L. Reduced Acute Bowel Toxicity in Patients Treated with Intensity-Modulated Radiotherapy for Rectal Cancer. Int. J. Radiat. Oncol. 2012, 82, 1981-1987. [CrossRef] [PubMed]

13. Deville, C.; Both, S.; Hwang, W.-T.; Tochner, Z.; Vapiwala, N. Clinical toxicities and dosimetric parameters after whole-pelvis versus prostate-only intensity-modulated radiation therapy for prostate cancer. Int. J. Radiat. Oncol. Biol. Phys. 2010, 78, 763-772. [CrossRef] [PubMed]

14. Deville, C.; Vapiwala, N.; Hwang, W.-T.; Lin, H.; Ad, V.B.; Tochner, Z.; Both, S. Comparative toxicity and dosimetric pro-file of whole-pelvis versus prostate bed-only intensity-modulated radiation therapy after prostatectomy. Int. J. Radiat. Oncol. Biol. Phys. 2012, 82, 1389-1396. [CrossRef]

15. The, B.S.; Mai, W.Y.; Uhl, B.M.; Augspurger, M.E.; Grant, W.H., III; Lu, H.H.; Woo, S.Y.; Carpenter, L.S.; Chiu, J.K.; Butler, E.B. Intensity-modulated radiation therapy (IMRT) for prostate cancer with the use of a rectal balloon for prostate immobili-zationacute toxicity and dose-volume analysis. Int. J. Radiat. Oncol. Biol. Phys. 2001, 49, 705-712.

16. Both, S.; Bui, V.; Hwang, W.-T.; Tan, K.-S.; Schaer, M.; Tochner, Z.; Vapiwala, N. Acute gastrointestinal and genitourinary toxicity of image-guided intensity modulated radiation therapy for prostate cancer using a daily water-filled endorectal balloon. Radiat. Oncol. 2012, 7, 76. [CrossRef]

17. Klopp, A.H.; Yeung, A.R.; Deshmukh, S.; Gil, K.M.; Wenzel, L.; Westin, S.N.; Gifford, K.; Gaffney, D.K.; Small, W., Jr.; Thompson, S.; et al. Patient-reported toxicity during pelvic intensity-modulated ra-diation therapy: NRG Oncology-RTOG. J. Clin. Oncol. 2018, 36, 2538-2544. [CrossRef]

18. Holyoake, D.L.; Partridge, M.; Hawkins, M.A. Systematic review and meta-analysis of small bowel dose-volume and acute toxicity in conventionally-fractionated rectal cancer radiotherapy. Radiother. Oncol. 2019, 138, 38-44. [CrossRef]

19. Arbea, L.; Ramos, L.; Beunza, J.; Moreno, M.; Cambeiro, M.; MartinezMonge, R.; Aristu, J. Dosimetric predictors of gastrointestinal toxicity during intensity modulated radiation therapy concomitant with capecitabine and oxaliplatin for locally advanced rectal cancer. Int. J. Radiat. Oncol. Biol. Phys. 2012, 84, S795. [CrossRef]

20. Xu, B.; Guo, Y.; Chen, Y.; Lu, H.; Tang, T.; Yue, Z.; Guan, G.; Chi, P.; Lin, C. Is the irradiated small bowel volume still a pre-dictor for acute lower gastrointestinal toxicity during preoperative concurrent chemo-radiotherapy for rectal cancer when using intensity-modulated radiation therapy? Radiat. Oncol. 2015, 10, 257. [CrossRef]

21. Peeters, S.T.; Hoogeman, M.S.; Heemsbergen, W.D.; Slot, A.; Tabak, H.; Koper, P.C.; Lebesque, J.V. Volume and hormonal effects for acute side effects of rectum and bladder during conformal radiotherapy for prostate cancer. Int. J. Radiat. Oncol. 2005, 63, 1142-1152. [CrossRef] [PubMed]

22. Hall, W.A.; Colbert, L.; Nickleach, D.; Shelton, J.; Marcus, D.M.; Switchenko, J.; Rossi, P.J.; Godette, K.; Cooper, S.; Jani, A.B. Reduced acute toxicity associated with the use of volumetric modulated arc therapy for the treatment of adenocarcinoma of the prostate. Pr. Radiat. Oncol. 2013, 3, e157-e164. [CrossRef] [PubMed]

23. Dias, R.S.; Giordani, A.J.; Souhami, L.; Segreto, R.A.; Segreto, H.R.C. Rectal planning risk volume correlation with acute and late toxicity in 3-dimensional conformal radiation therapy for prostate cancer. Technol. Cancer Res. Treat. 2011, 10, 585-590. [CrossRef] [PubMed]

24. Engels, B.; De Ridder, M.; Tournel, K.; Sermeus, A.; De Coninck, P.; Verellen, D.; Storme, G.A. Preoperative helical tomo-therapy and megavoltage computed tomography for rectal cancer: Impact on the irradiated volume of small bowel. Int. J. Radiat. Oncol. Biol. Phys. 2009, 74, 1476-1480. [CrossRef]

25. Chung, H.T.; Xia, P.; Chan, L.W.; Park-Somers, E.; Roach, M. Does Image-Guided Radiotherapy Improve Toxicity Profile in Whole Pelvic-Treated High-Risk Prostate Cancer? Comparison Between IG-IMRT and IMRT. Int. J. Radiat. Oncol. 2009, 73, 53-60. [CrossRef]

26. Paydar, I.; Cyr, R.A.; Yung, T.M.; Lei, S.; Collins, B.T.; Chen, L.N.; Suy, S.; Dritschilo, A.; Lynch, J.H.; Collins, S.P. Proctitis 1 Week after Stereotactic Body Radiation Therapy for Prostate Cancer: Implications for Clinical Trial Design. Front. Oncol. 2016,6 , 167. [CrossRef]

27. Hwang, M.; Mayeda, M.; Liz, M.; Goode-Marshall, B.; Gonzalez, L.; Elliston, C.D.; Spina, C.S.; Padilla, O.A.; Wenske, S.; Deutsch, I. Stereotactic body radiotherapy with periprostatic hydrogel spacer for localized prostate cancer: Toxicity profile and early oncologic outcomes. Radiat. Oncol. 2019, 14, 1-9. [CrossRef]

28. de Boer, P.; van de Schoot, A.J.; Westerveld, H.; Smit, M.; Buist, M.R.; Bel, A.; Rasch, C.R.N.; Stalpers, L.J.A. Target tai-loring and proton beam therapy to reduce small bowel dose in cervical cancer radiotherapy: A comparison of benefits. Strahlenther. Onkol. 2018, 194, 255-263. [CrossRef] 